\title{
Nine newly discovered bright low-redshift quasars from RASS
}

\author{
D.W. Xu ${ }^{1}$, J.Y. Wei ${ }^{1,2}$, X.Y. Dong ${ }^{1}$, and J.Y. $\mathbf{H u}^{1,2}$ \\ 1 Beijing Astronomical Observatory, Chinese Academy of Sciences, Datun Rd., \#20A, Chaoyang District, Beijing 100012, \\ PR China \\ e-mail: dwxu@class1.bao.ac.cn \\ ${ }^{2}$ United Laboratory of Optical Astronomy, Chinese Academy of Sciences, PR China
}

Received May 19; accepted August 24, 1998

\begin{abstract}
By using the CCD detector and Cassegrain spectrograph of the $2.16 \mathrm{~m}$ telescope at Beijing Astronomical Observatory, we are carrying out optical identifications of the X-ray sources selected from the ROSAT All-Sky Survey Bright Source Catalogue. We present here the discovery of 9 bright low-redshift quasars.
\end{abstract}

Key words: quasars: general — surveys - X-rays: galaxies

\section{Introduction}

During the ROSAT All-Sky Survey (RASS) about 80000 $\mathrm{X}$-ray sources with a detection likelihood $\geq 10$ were found (Voges 1992; Voges et al. 1994; Voges et al. 1996b, 1997), from which 18811 sources were compiled in the ROSAT Bright Source Catalogue (RASS-BSC) (Voges et al. 1996a,b; Voges et al. 1997). Most of them are newly discovered X-ray sources and more than $65 \%$ of the objects were previously unknown (Brinkmann et al. 1995; Bade et al. 1998). The optical spectroscopic studies of these RASS sources are essential for understanding them more clearly. We are performing a program to identify unknown RASS sources at high galactic latitude $\left(|b| \geq 20^{\circ}\right)$ in the northern hemisphere $\left(\delta \geq 3^{\circ}\right)$ and whose likely optical counterparts have $R$ magnitudes between 13.5 and 16.5. About 60 quasars were identified independently through low dispersion spectroscopic observations in 1996 and 1997 (Wei et al. 1996, 1997). In this paper we report on the discovery of 9 bright low-redshift quasars (hereafter BLRQs) with $B$ magnitude equal to or less than 17.0.

In Sect. 2, the sample selection is introduced. The observations and results are presented in Sect. 3 .

$\overline{\text { Send offprint requests to }}$ : D.W. Xu
Table 1. Journal of observations

\begin{tabular}{llrc}
\hline \hline ROSAT name & Obs. Date & $\begin{array}{r}\text { Exposure } \\
(\mathrm{sec})\end{array}$ & $\begin{array}{c}\text { dispersion } \\
{\left[\AA \mathrm{mm}^{-1}\right]}\end{array}$ \\
\hline 1RXS J071339.7+382043 & Jan. 28 1997 & 800 & 200 \\
1RXS J073308.7+455511 & Jan. 2 1997 & 1200 & 200 \\
1RXS J092748.9+403838 & Jan. 1 1997 & 3100 & 200 \\
1RXS J101031.1+255959 & Dec. 19 1996 & 1000 & 200 \\
1RXS J104111.5+282812 & Jan. 6 1997 & 1800 & 200 \\
1RXS J111754.9+263413 & Jan. 30 1997 & 1800 & 200 \\
1RXS J120347.5+520748 & Dec. 19 1996 & 600 & 200 \\
1RXS J120423.7+433059 & Jan. 2 1997 & 2100 & 200 \\
1RXS J122542.3+205503 & Dec. 18 1996 & 1200 & 200 \\
\hline
\end{tabular}

Table 2. X-ray characteristic of the new quasars

\begin{tabular}{lccrr}
\hline \hline ROSAT name & $\begin{array}{c}\text { Error radius } \\
\text { (arcsec) }\end{array}$ & $\begin{array}{c}\text { Cnt rate } \\
\text { (cts/s) }\end{array}$ & HR1 & HR2 \\
\hline 1RXS J071339.7+382043 & 8 & 0.120 & 0.75 & 0.12 \\
1RXS J073308.7+455511 & 8 & 0.273 & 0.83 & 0.14 \\
1RXS J092748.9+403838 & 9 & 0.190 & -0.25 & -0.06 \\
1RXS J101031.1 + 255959 & 14 & 0.054 & -0.57 & 0.38 \\
1RXS J104111.5 +282812 & 8 & 0.221 & -0.26 & 0.15 \\
1RXS J111754.9+263413 & 12 & 0.068 & -0.85 & 0.00 \\
1RXS J120347.5+520748 & 7 & 0.361 & -0.36 & 0.08 \\
1RXS J120423.7+433059 & 9 & 0.091 & -0.48 & -0.80 \\
1RXS J122542.3+205503 & 10 & 0.329 & -0.63 & -0.20 \\
\hline
\end{tabular}

\section{Sample selection}

Our sample is selected from RASS-BSC according to the following criteria:

1. Declination $\delta \geq 3^{\circ}$.

2. Galactic latitude $|b| \geq 20^{\circ}$.

3. Optical counterparts within a circle with radius $r=$ $r^{\prime}+5^{\prime \prime}$, where $r^{\prime}$ is the RASS position error given by Voges et al. (1996b). 



Fig. 1. Spectra of the new quasars 
Table 3. Optical properties of the new quasars

\begin{tabular}{|c|c|c|c|c|c|c|c|c|}
\hline ROSAT name & $\begin{array}{c}\mathrm{RA} \\
(2000)\end{array}$ & $\begin{array}{l}\text { DEC } \\
(2000)\end{array}$ & $\begin{array}{c}D_{\mathrm{o}-\mathrm{x}} \\
(\operatorname{arcsec})\end{array}$ & $\bar{R}$ & $\bar{B}$ & $\bar{z}$ & 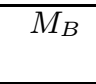 & Comments \\
\hline 1RXS J071339.7+382043 & 071340.30 & 382039.7 & 9.6 & 14.8 & 16.0 & 0.123 & -23 & $\begin{array}{l}\mathrm{H}_{\delta}, \mathrm{H}_{\gamma}, \mathrm{FeII}, \mathrm{H}_{\beta}, \mathrm{HeI}, \mathrm{H}_{\alpha} \\
{[\mathrm{OII}],[\mathrm{NeIII}],[\mathrm{OIII}]}\end{array}$ \\
\hline 1RXS J073308.7+455511 & 073309.27 & 455505.6 & 10.1 & 15.5 & 16.2 & 0.142 & -23.5 & $\begin{array}{l}\mathrm{H}_{\delta}, \mathrm{H}_{\gamma}, \mathrm{FeII}, \mathrm{H}_{\beta}, \mathrm{HeI}, \mathrm{H}_{\alpha} \\
{[\mathrm{OII}],[\mathrm{NeIII}],[\mathrm{OIII}]}\end{array}$ \\
\hline 1RXS J092748.9+403838 & 092749.07 & 403831.7 & 6.8 & 16.2 & 16.4 & 0.423 & -25.7 & $\mathrm{MgII}, \mathrm{H}_{\gamma}, \mathrm{H}_{\beta},[\mathrm{OII}],[\mathrm{OIII}]$ \\
\hline 1RXS J101031.1+255959 & 101030.55 & 255948.7 & 13.2 & 16.3 & 16.7 & 0.512 & -25.8 & $\begin{array}{l}\mathrm{MgII}, \mathrm{H}_{\delta}, \mathrm{H}_{\gamma}, \mathrm{H}_{\beta} \\
{[\mathrm{OII}],[\mathrm{NeIII}],[\mathrm{OIII}]}\end{array}$ \\
\hline 1RXS J104111.5+282812 & 104111.95 & 282804.7 & 7.7 & 16.1 & 16.8 & 0.210 & -23.8 & $\begin{array}{l}\mathrm{H}_{\delta}, \mathrm{H}_{\gamma}, \mathrm{FeII}, \mathrm{H}_{\beta}, \mathrm{HeI}, \mathrm{H}_{\alpha} \\
{[\mathrm{NeIII}],[\mathrm{OIII}]}\end{array}$ \\
\hline 1RXS J111754.9+263413 & 111754.33 & 263416.4 & 9.2 & 16.1 & 16.9 & 0.422 & -25.2 & $\mathrm{MgII}, \mathrm{H}_{\gamma}, \mathrm{H}_{\beta},[\mathrm{OIII}]$ \\
\hline 1RXS J120347.5+520748 & 120347.71 & 520749.5 & 3.5 & 16.0 & 16.8 & 0.177 & -23.4 & $\begin{array}{l}\mathrm{H}_{\delta}, \mathrm{H}_{\gamma}, \mathrm{HeII}, \mathrm{H}_{\beta}, \mathrm{H}_{\alpha}, \\
{[\mathrm{NeV}],[\mathrm{NeIII}],[\mathrm{OIII}]}\end{array}$ \\
\hline 1RXS J120 & 120424 . & 433 & 5.5 & 15.6 & 16.5 & 0.663 & -26.6 & $\mathrm{MgII}, \mathrm{H}_{\gamma},[\mathrm{OII}]$ \\
\hline 1RXS J122542.3+205503 & 122541.89 & 205503.7 & 6.2 & 16.1 & 16.5 & 0.334 & -25.1 & $\mathrm{H}_{\gamma}, \mathrm{H}_{\beta},[\mathrm{OIII}]$ \\
\hline
\end{tabular}

4. Optical counterparts with $R$ magnitudes between 13.5 and 16.5 , where $R$ magnitude was derived from USNO$\mathrm{A} 1.0^{1}$.

5. $\log C \geq-0.4 R+4.9$.

where $C$ is the X-ray count rate of RASS source and $R$ is the $R$ magnitude of possible counterpart. The results of the EMSS (Stocke et al. 1991) have shown that different classes of X-ray sources represent different narrow ranges in the X-ray-to-optical flux ratios. These bounds mean the X-ray sources with Galactic and extragalactic counterparts can be separated at high confidence level prior to any optical spectroscopy (Maccacaro et al. 1988). However, X-ray flux is difficult to evaluate before optical identification. In spite of the absorption by the column density $N_{\mathrm{H}}$ of cold material X-ray count rate is roughly proportional to $\mathrm{X}$-ray flux, so X-ray flux in the X-ray-to-optical flux ratio criterion can be replaced by $\mathrm{X}$-ray count rate, i.e., $\log \left(f_{\mathrm{X}} / f_{\mathrm{O}}\right) \propto \log C+0.4 R+$ constant, where we use the $R$ magnitude flux to represent the optical flux. According to our statistical analysis of known RASS-BSC X-ray sources, we found there is an apparent gap between Galactic stars and extragalactic objects. Emission line AGNs concentrate on the region: $\log C \geq-0.4 R+4.9$. Thus choosing $\log C \geq-0.4 R+4.9$ as preselecting AGNs criterion would be efficient. Details of the criterion construction have been described in Cao et al. (1998);

6. No association with objects in the Galactic and extragalactic catalogues compiled in the SIMBAD and NED database.

\footnotetext{
1 USNO-A1.0, 1996, CD-ROM version, U.S. Naval Observatory.
}

\section{Spectroscopic observations and results}

The spectroscopic observations were made in several observing runs in 1996 and 1997 with the $2.16 \mathrm{~m}$ telescope of the United Laboratory of Optical Astronomy, Chinese Academy of Sciences at Xinglong Station, Beijing Astronomical Observatory (BAO). The spectra were obtained with the OMR spectrograph equipped with a Tektronix CCD $(1024 \times 1024$ pixels with 24 microns per pixel) at a dispersion of $200 \AA \mathrm{mm}^{-1}$ or $400 \AA \mathrm{mm}^{-1}$. The $400 \AA \mathrm{mm}^{-1}$ grating was not used if one believes Table 1. The spectral coverage was $3800-8200 \AA$. The journal of observations is given in Table 1.

The data reduction was performed by using the IRAF program package. The CCD reductions included bias subtraction, flatfield correction and cosmic-ray removal. Spectra of a He-Ne-Ar or Fe-Ar lamp were taken to get an absolute wavelength scale. The flux calibration was derived with 2 to 3 observations of KPNO IIDS standard Stars (Strom 1979) per night. The atmospheric extinction was corrected by using the mean extinction coefficients of Xinglong Station, which were measured by BATC multicolor survey (Yan 1995).

The X-ray characteristics and optical properties of the 9 BLRQs are summarized in Tables 2 and 3, and their spectra are shown in Fig. 1. Position error radius, count rates, hardness ratios 1 \& 2 listed in Table 2 are from RASS-BSC. Hardness ratios are defined as:

$H R 1=\frac{H-S}{H+S}$

$H R 2=\frac{H_{1}-H_{2}}{H_{1}+H_{2}}$

where $H, S, H_{1}$, and $H_{2}$ are the count rates in the hard $H$-band $(0.4-2.4 \mathrm{keV})$, the soft $S$-band $(0.07-0.4 \mathrm{keV})$, the hard $H_{1}$-band $(0.4-1.0 \mathrm{keV})$ and the hard $H_{2}$-band $(1.0-2.4 \mathrm{keV})$ respectively. The coordinates given in 
Table 3 were obtained from USNO-A1.0. We applied these coordinates to the Digitized Sky Survey ${ }^{2}$ and did not find any ambiguous identifications of the objects, so we do not give the finding charts of the 9 BLRQs. The average redshifts were usually determined from two or more emission lines. The $B$ and $R$ magnitudes were obtained from USNO-A1.0 and have an accuracy between 0.25 and 0.40 magnitudes depending on the declination (Monet et al. 1996). The absolute $B$ magnitudes were calculated by the same formula used by Véron-Cetty \& Véron (1996) under the assumption of $H_{0}=50 \mathrm{~km} \mathrm{~s}^{-1} \mathrm{Mpc}^{-1}$ and $q_{0}=0$. The $D_{\mathrm{x}-\text { opt }}$ listed in Table 3 is the angular separation between the X-ray centroïd and the optical countpart in arcseconds.

Acknowledgements. Special thanks to Dr. Qiu Yulei and Dr. Jiang Biwei for their hard work of English revision of this paper. We are grateful to Miss Cao Li for her kindly help with data reduction. We also thank Mr. Yan Haojing for providing the extinction data of Xinglong Station. This work is partly supported by the Pandeng Project of the Chinese Scientific Committee and the Chinese National Natural Science Foundation.

\section{References}

Bade N., Engels D., Voges W., et al., 1998, A\&AS 127, 145

Brinkmann W., Siebert J., Reich W., et al., 1995, A\&AS 109, 147

Cao L., Wei J.Y., Hu J.Y., 1998 (to be submitted A\&A)

Maccacaro T., Gioia I., Wolter A., et al., 1988, ApJ 326, 680

Monet D.G., et al., 1996, USNO-A1.0. Washington DC, USNO

Stocke J.T., Morris S.L., Gioia I.M., et al., 1991, ApJS 76, 813

Strom K.M., 1979, Kitt Peak National Observatory IIDS Standard Star Manual

Véron-Cetty M.-P., Véron P., 1996, ESO Scientific Report, No. 17

Voges W., 1992, in Proceedings of the ISY Conference "Space Sciences", ESA ISY-3, ESA Publications, p. 9

Voges W., Gruber R., Haberl F., et al., 1994, ROSAT NEWS No. 392

Voges W., Boller Th., Dennerl K., et al., 1996a, MPE-Report 263, 637

Voges W., Aschenbach B., Boller Th., et al., 1996b, IAU Circ. 6420

Voges W., Aschenbach B., Boller Th., et al., 1997 (to be submitted A\&AS)

Wei J.Y., Cao L., Hu J.Y., et al., 1996, in Proceedings of IAU Colloquium 159, p. 425

Wei J.Y., Cao L., Xu D.W., et al., 1997, Acta Astrophys. Sin. 17,107

Yan H.J., 1995 (private communication)

\footnotetext{
2 The Digitized Sky Survey, 1994, CD-ROM Version, Space
} Telescope Science Institute. 